\title{
Multi-Depot Capacitated Vehicle Routing Problem by Using Stable Marriage and K-Means Clustering to Minimize Number of Unserved Customers and Total Travel Distance
}

\author{
Purba Daru Kusuma $^{1 *} \quad$ Meta Kallista $^{1}$ \\ ${ }^{1}$ Computer Engineering, Telkom University, Indonesia \\ * Corresponding author's Email: purbodaru@telkomuniversity.ac.id
}

\begin{abstract}
Multi-depot capacitated vehicle routing problem is a derivative of vehicle routing problem and a wellknown area that has been studied widely with various applications and constraints. Many studies in it focused on minimizing cost and distance with assumption that all nodes are visited. Ironically, number of unserved customers (nodes) in a single trip was rarely explored and often ignored. Based on this problem, this work aims to minimize the total travel distance and the number of unserved customers in single routing cycle. The solution is developed by combining the stable marriage algorithm and k-means clustering in the clustering process. The nearest neighbour algorithm is used in the routing process. This work proposes two contributions. The first is the usage of the stable marriage and k-means clustering. The second is concerning the predetermined distribution of the vehicles in the solution to reduce the number of unserved customers. In the simulation, this proposed model is compared with the hybrid evolutionary algorithm (HEA), partition-based algorithm-nearest neighbour algorithm (PBA-NN), genetic algorithm-nearest neighbour algorithm (GA-NN) and simulated annealing algorithm (SA). Based on the simulation result, it is found that the proposed model performs moderately as a trade-off between the SA and PBA-NN. In the number of unserved customers aspect, when the number of customers is low, the proposed model creates 71 percent lower than the PBA-NN model. Meanwhile, when the number of customers is high, the proposed model creates 73 percent lower than the PBA-NN model. In the total travel distance aspect, when the number of customers is low (50 persons), the proposed model creates 63 percent higher than the GA-NN model and 48 percent lower than the SA model. Meanwhile, when the number of customers is high (100 customers), the proposed model creates 52 percent higher than the PBA-NN model and 54 percent lower than the SA model.
\end{abstract}

Keywords: Capacitated vehicle routing problem, Multi depots, Stable marriage, K-means clustering, Nearest neighbour, Supply chain management.

\section{Introduction}

Vehicle routing problem (VRP) is a popular object that has been studied widely. The VRP is a derivative of the traveling salesman problem (TSP) [1]. The general concept is there is a vehicle that departs from and returns to the same node [2]. During its journey, the vehicle must visit all nodes where each node must be visited once [3]. The main goal is minimizing the total travel distance from its departure to its return $[4,5]$.

The VRP has been studied widely with many derivatives, circumstances, and constraints. Several specific VRP studies are as follows. The multi-depot vehicle routing problem (MDVRP) is a VRP variant where there are several depots and the vehicles in the system are distributed among depots [6]. The capacitated vehicle routing problem (CVRP) is a VRP variant where there is limitation or constraint in the vehicle capacity so that the vehicle can load only less than or equal to its capacity [7]. Green vehicle routing problem (GVRP) is a VRP variant that concerns with reducing pollution or carbon emission due to the traveling process $[8,9]$. In it, refueling process during the routing process is permitted. The 
electric vehicle routing problem (EVRP) is a VRP variant that uses electric vehicles $[10,11]$. The special characteristic of the electric vehicle is its limited range compared with the conventional combustion vehicle [10]. It makes the electric vehicle must be recharged during the routing process so that additional charging stations are augmented in the system [10].

In common, the goal of the vehicle routing problem is minimizing the total travel distance [12]. Moreover, several goals can be derived from this main goal, due to the specific objective of the study. Some VRP studies aimed to minimize total cost [13], where the cost structure is constructed from the fixed cost and the variable cost [14]. In the GVRP study, the goal is minimizing pollution [8]. The other goal is improving service level [15] by visiting customers inside the time window. Several VRP studies have the objective of minimizing the number of dispatched vehicles [10]. In some studies, a vehicle can have multiple trips to execute all orders [16].

Ironically, studies in VRP that their objective is minimizing the number of unserved customers, are rare. Despite that, the unserved customers aspect is also an important factor that affects the customer satisfaction. The minimization of the number of unserved customers improves the service level.

In this work, the multi-depot capacitated vehicle routing problem is proposed. The vehicles are distributed among predetermined depots. The vehicles' role is collecting products from customers. The demand is static so that it is predetermined before the routing process starts. It is different from the dynamic demand where the customers are known but the requested quantity is unknown [12] or the stochastic demand where there are two types of customers: the predetermined customers and customers that exist during the routing process [14]. In this work, unserved customers are allowed. This work has two objectives. The first objective is to minimize the total travel distance. The second objective is to minimize the number of unserved customers in a single routing cycle.

This work is developed by using stable marriage algorithm, K-means clustering, and nearest neighbor algorithm. Stable marriage algorithm is chosen in the clustering process due to its characteristic in matching two parties with the highest possible preference [20]. This algorithm has been implemented in many areas, such as school admission [21], hospital-residence [22], and online taxi dispatching system [23, 24]. Ironically, this stable marriage algorithm is rare to be used in the vehicle routing problem. $\mathrm{K}$-means clustering is also a popular clustering technique that has been used widely in many areas, such as education [25], distributed computing [26], and so on. The nearest neighbor algorithm is a proven lightweight routing algorithm that has been used and combined in several VRP studies $[8,9]$.

Contributions of this work are as follows.

(1) This work develops a new CVRP model by combining stable marriage algorithm, K-means clustering, and nearest neighbor algorithm.

(2) This work proposes unserved customers in a single routing cycle as an important aspect in CVRP besides total travel distance.

The remainder of this paper is organized as follows. In the second section, several works in CVRP studies are explored, include the used technique and scenario. In the third section, the proposed model, that consists of algorithm and the mathematical model, is explained. In the fourth section, the simulation that is conducted to evaluate the performance of the model, the simulation result, and the research findings are discussed. In the fifth section, the work is concluded, and the future research potential is explored.

\section{Related works}

Capacitated vehicle routing problem (CVRP) has been studied widely. Every CVRP study tried to solve a specific problem, used specific solutions, and was implemented in some specific circumstances. It is because there is not any generic solution that is optimal in solving all problems [1]. Besides, every CVRP study had specific objectives.

CVRP can be called as a TSP with capacity constraint [1]. CVRP can also be modeled as a graph that consists of a set of nodes that represents a set of customers [1]. On the other side, there is a node that represents a depot (if it is a single depot scenario) where every vehicle departs from and return to [1]. Each customer is served by one vehicle [1]. Below are several related studies in CVRP with their specific circumstances and solutions.

Ahmed and sun [1] proposed CVRP with a single depot scenario. Its objective was to find the minimum total cost, minimum travel distance, and service time. Besides being restricted by the capacity, the vehicle is also restricted by the maximum travel time. This work implemented static demand where the demand is pre-determined. This work implemented bilayer local search-based particle swarm optimization (BLS-PLO). The first layer was applied as a local search for any iteration. The second layer acted as a pool of best particles along 
with generations.

Cuevas, Omar, Morales, Flores, and Vazquez [13] developed CVRP model for carrier companies. This work implemented a single depot scenario. Rather than distributing products to customers, the vehicles collect products from customers to be delivered to specific a warehouse. Every vehicle was identical. The CVRP problem was modeled by using integer programming. Its objective was to minimize the total cost which consists of the travel cost and the returning cost.

Normasari, Bachtiyar, and Sukoyo [4] developed GVRP problem. Its objective was aiming at both economic and environmental aspects. The problem was modeled in a mixed integer linear programming (MILP) which consisted of an objective function and several mathematical constraints. This work implemented a single depot scenario. Each vehicle made a single trip. The maximum travel time constraint was applied.

Okulewicz and Mandziuk [12] developed multidepot dynamic VRP model. All vehicles were identical in both capacity and speed. The other constraint was the depot working hour so that every vehicle must return to the depot during its operational hour. The customers had to be served in a certain time window. The euclidean distance was used to measure the travel distance. To solve the dynamic demand, routing and optimization ran in every discrete time slice. The objective was minimizing travel distance within time constraints and depot working hours.

Lin, Zhou, and Wolfson [10] proposed a model for EVRP. They stated that the problem of electric vehicles was their limited operational range so that it must visit the charging station during their daily operation. During operation, a vehicle could visit charging station multiple times. A charging station could be visited by multiple vehicles. A single depot scenario was implemented. The goal was minimizing travel time cost, energy cost (battery charging cost), charging waiting time, and number of dispatched vehicles.

Shao, Guan, Ran, He, and Bi [11] also developed model for EVRP problem. This work used genetic algorithm (GA) and dynamic Dijkstra algorithm to find the shortest path between two adjacent nodes. Rather than constant speed, the travel time fluctuated due to the dynamic traffic environment. This work used mixed integer linear programming (MILP) to formulate the problem. Its objective was minimizing cost that consisted of vehicle fixed cost, travel cost, penalty cost, and charging costs.

Jahangir, Mohammadi, Pasandideh, and Nobari [19] proposed CVRP model where a single depot serves and distributes a single product to its customers. The vehicles were heterogeneous. Backorders are allowed. Customers were served only when their inventory level is below the minimum inventory level. This work used mixed integer linear programming (MILP) to formulate the problem. Its objective was minimizing transportation costs and inventory costs. This work used two metaheuristic algorithms: genetic algorithm and invasive weed optimization. Although multiple trips were allowed, a vehicle was designed to trip once.

Qiao, Tao, Wu, Yu, and Zhang [17] developed a CVRP model to be implemented in the municipal solid waste collection system. The vehicle departed from the depot with an empty load, visited and collected waste during its trip, unloaded the waste at the disposal facility, and then returned to the depot. A vehicle might take multiple trips. Every vehicle was identical. Its objective was minimizing cost that consisted of the vehicle fixed cost, fuel consumption cost, carbon emission cost, and penalty cost. This work used the combined PSO and TS algorithm.

Alhujaylan and Hosny [18] developed a VRP model that combined pickup and delivery roles. It implemented a single depot scenario. It used greedy randomized adaptive search (GRASP). It implemented a static VRP problem so that all problems are known in advance. Besides capacity constraint, there was a travel time constraint so that a vehicle might not exceed the maximum daily travel time. This model also allowed unserved customers due to the company's limited resources.

Khaoula, Youssef, and Ghizlane [5] also developed an MDVRP model for pickup and delivery scenarios. In it, the vehicles were identical. The role of vehicles was distributing products to customers and picking up the return products from customers. Both depots and vehicles had capacity constraint. This work used genetic algorithm for the routing process. Meanwhile, K-nearest depot was used to assign customers to its nearest depot.

Ouaddi, Benadada, and Mhada [16] developed a DVRP model by using ACO. In it, a vehicle could return to the depot after the operational time but had to be on the same day. Due to the dynamic demand, there were several planning periods in one day. Orders that arrived in a current planning period would be executed in the next planning period. A vehicle might have multiple tours due to many orders and a limited number of vehicles.

Ge, Zhu, and Jin [14] developed an EVRP model for solving the stochastic demand and dynamic demand. In it, there were two tiers of warehouses: the central warehouse, and the front warehouses. This work used mixed integer linear programming 
(MILP) to formulate the problem. The distribution planning consisted of two cycles. The first cycle was the planning that the quantity was determined based on the historical data. Meanwhile, the unserved demand that arrived during the planning period would be executed in the second cycle. It meant that a vehicle might have multiple trips.

There are several notes due to the works of literature above. First, most of the studies in VRP focused on minimizing travel distance which is a part of the total cost. Although several studies allowed multiple trips for a vehicle, it should be minimized so that a single trip policy was prioritized. Ironically, studies with the objective were to minimize the unserved requests in the single trip, were rare.

Second, in general, the capacitated vehicle routing problem is solved by using one of these approaches: metaheuristic, deterministic, or combination between metaheuristic and deterministic. The nearest neighbor technique is the most common deterministic technique that is used in the CVRP. On the other side, the stable marriage is not a popular solution in the CVRP although it was widely used in the taxi dispatching system and other optimization works.

Based on these notes, there are two unique positions of this work, compared with the existing works. The first is the objective in minimizing the unserved requests in a single trip execution. The second is the usage of the stable marriage, which is combined with another method, to solve the multi depot capacitated vehicle routing problem.

\section{Model}

As a multi-depot capacitated vehicle routing problem (MDCVRP), there are several rules and assumptions that are used in this model. These rules and assumptions are as follows.

- The number of vehicles and their depot are predetermined [16].

- The demand is static. It means that the number of customers and their request order is known before the routing process, and it does not change during the routing process [18].

- The vehicles are identical in capacity and speed [12].

- The vehicles collect the customers' product and then delivers it to the depot [17].

- Vehicle's load cannot surpass its capacity constraint [18].

- Every vehicle departs from and returns to the same depot [3].

- The depot capacity is the accumulation of the capacity of the vehicles that are docked at this depot.

- Every vehicle has a single trip [4].

- Euclidean distance is used to measure the distance between two points [12]

The proposed model is developed by using clustering-first routing-second approach [7]. It means that in the beginning, the customers are clustered first so that the customers in the same cluster will be executed by the same vehicle. The clustering process consists of two steps. The first step is clustering the customers to the nearest available depots. The stable marriage algorithm is used in this first step. The second step is clustering the customers in the same depot. The k-means clustering is used in this second step. After the clustering process, the next process is the routing process. In this work, the routing process uses nearest neighbor algorithm. Notations that are used in this work are as follows.

$c \quad$ customer

$d \quad$ depot

$v \quad$ vehicle

$e \quad$ centroid / cluster

$d_{s} \quad$ selected depot

$c_{s} \quad$ selected customers

$c_{n} \quad$ next customer

$D_{s u} \quad$ set of depots whom the customer has submitted to

$C_{c u} \quad$ set of customers that has active proposal to the depot

$q_{c} \quad$ customer's requested capacity

$q_{d} \quad$ depot capacity

$q_{v} \quad$ vehicle capacity

$q_{\text {vnow }}$ vehicle's current load

$q_{g} \quad$ gap quantity

$l_{v d} \quad$ link between vehicle and depot, 1 means that the vehicle is linked to the depot and 0 means that the vehicle is not linked to the depot

$l_{c d} \quad$ link between customer and depot, 1 means that the customer is linked to the depot and 0 means that the customer is not linked to the depot

$l_{v e} \quad$ link between vehicle and cluster, 1 means that the vehicle is linked to the cluster and 0 means that the vehicle is not linked to the cluster

$l_{c e} \quad$ link between customer and cluster, 1 means that the customer is linked to the cluster and 0 means that the customer is not linked to the cluster

$t \quad$ time

$n(c) \quad$ number of customers

$s_{c} \quad$ customer status, $1=$ served and $0=$ unserved

$v_{s} \quad$ selected vehicle 
In the first clustering, the stable marriage algorithm is used. This algorithm was founded by Gale and Shapley [20]. It was used in the matching process between the equal number of men and women based on their preference [20]. Then, still in their work, this algorithm was used in the school admission system [20].

In this work, the customers become the proposal submitters and the depots become the proposals evaluators. It means the system is customers dominant because the customers have a better opportunity to match with their most preferred depots [27]. The customer's satisfaction decreases only when the depot rejects its proposal. On the other side, the depot improves its satisfaction only if it declines its weakest current proposal and accepts the new incoming proposal. In this work, the stable marriage algorithm is defined as follows [20].

1. In the beginning, all customers submit proposal to their most preferred depot.

2. Then, the depots sort the proposals based on their preference. The depots accept proposals based on this rank and the depots' capacity. Depots accept their most preferred proposals if only their capacity can handle the requested orders. The rest of the proposals are rejected. Customers whose proposal is accepted become temporary engaged with their targeted depot. Customers whose proposal are declined are still unengaged.

3. The unengaged customers can resubmit new proposal to their most current preferred depots. The depots whom the customer have submitted a proposal in the previous time are excluded.

4. The second and the third steps are repeated until the matching process stable. The matching process is stable when there is not any change in the acceptance/rejection list.

The only aspect that is used as a preference is the distance. The customers prioritize the nearest depot to it. On the other side, the depot also prioritizes the nearest customers to it. This mechanism is formalized by using Eq. (1) to Eq. (3). This first clustering is illustrated in Fig. 1.

$$
\begin{aligned}
& d_{s}=\min (\|d-c\|), d \notin D_{s u} \\
& c_{s}=\operatorname{minsort}(\|d-c\|) \wedge \sum q_{c} \leq q_{d}, c \in C_{c u} \\
& q_{d}=\sum\left(q_{v} \cdot l_{v d}\right)
\end{aligned}
$$

The explanation of Eq. (1) to Eq. (3) is as follows. Eq. (1) shows that the customer submits to the depot

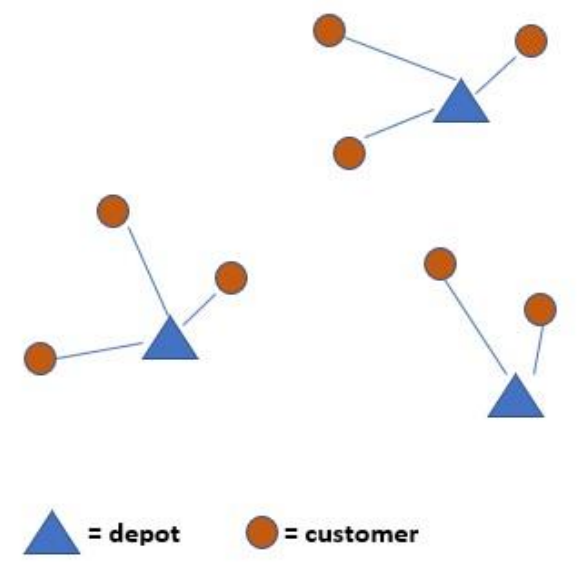

Figure. 1 Customer to depot clustering

that is nearest to it and it has not submitted to this depot in the previous time. Eq. (2) shows that the depot accepts customers that are nearest to their active proposal is submitted to it and its capacity can fulfill their requested order. Eq. (3) shows that the depot capacity is the accumulation of the capacity of the trucks that are docked at this depot.

The second clustering is intra-depot clustering. It uses k-means clustering. The goal of k-means clustering is to minimize the total distance among members in the clusters [28]. The number of clusters or centroids in every depot is equal to the number of trucks that are docked at this depot. This k-means clustering is formalized by using Eq. (4) to Eq. (6). Based on [28], the k-means clustering algorithm in every depot that is used in this work is as follows. The illustration is shown in Fig. 2.

1. In the beginning, the centroids are located randomly among customers in the depot.

2. Then, every customer is linked to the nearest centroid.

3. Every centroid location is updated based on the average location of customers that are connected to it if the centroid has at least one member. If the centroid does not have any members, its new location is determined by using step 1.

4. Step 2 and step 3 are repeated until it converges. The convergence is achieved when there is not any cluster member shifting.

$$
\begin{aligned}
& e(0)=\operatorname{random}(c), \forall c, l_{c d}=1 \\
& l_{c e}=\left\{\begin{array}{c}
1, \min (\|c-e\|) \\
0, \text { else }
\end{array}\right. \\
& e(t)=\left\{\begin{array}{c}
\frac{\sum c}{n(c)}, n(c)>0 \\
\operatorname{random}(c), \forall c, l_{c d}=1
\end{array}, t>0\right.
\end{aligned}
$$




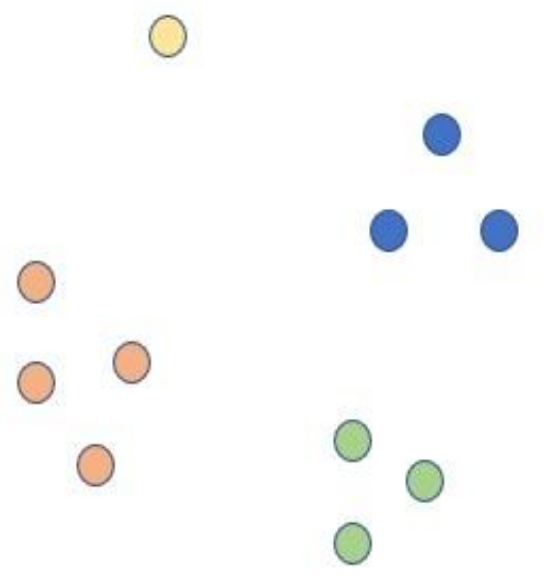

Figure. 2 Intra depot clustering

The explanation of Eq. (4) to Eq. (6) is as follows. Eq. (4) shows that the centroid initial position is randomized among the customers in the depot. Eq. (5) shows that the link between centroid and customer is 1 only if its location is the nearest. In other words, the customer is linked to the centroid that is nearest to it. Eq. (6) shows that the centroid's next location is the average location of its member only if it has at least one member. Else, its new location will be randomized among the customers location that are linked to the depot.

After the customers have been clustered, the next process is the routing process. The routing process is conducted by using nearest neighbor algorithm as it is also used in the previous works [8]. In general, in the nearest neighbor algorithm, the next destination is the nearest unvisited node [8].

In this work, the routing process is conducted in two steps. The first step is intra-cluster routing. In the first routing, every vehicle is attached to one cluster. The second step is non-cluster routing. The second routing is conducted if there exists any customer that has not been served during the first routing.

In the first routing, every vehicle in the depot will route customers that has the same cluster to the vehicles. The algorithm of the first cluster is as follows [8]. The illustration is shown in Fig. 3.

1. Vehicle starts from the depot with empty load.

2. Vehicle searches unserved customer in the cluster that is nearest to it and the summation between vehicle's current load and the customer's requested quantity does not surpass the vehicle capacity.

3. If this potential customer is found, then the vehicle goes to the customer and pickups the requested product.

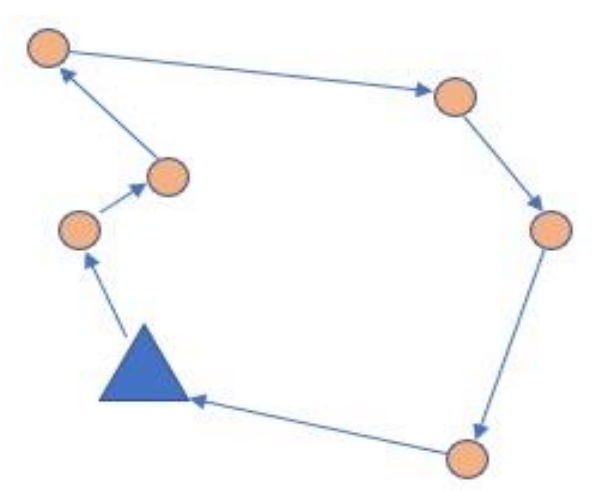

Figure. 3 Nearest neighbor routing illustration

4. Step 2 and step 3 are repeated until one of these two conditions occurs. The first condition is the vehicle cannot serve anymore because their current load is full or almost full so that there is not any customer in the cluster that can be served. The second condition is there is not any unserved customer in the cluster.

This algorithm is formalized by using Eq. (7) and Eq. (8). Eq. (7) shows that the next customer is selected if it is the nearest to the vehicle, it is the same cluster with the vehicle, the capacity constraint is obeyed, and its status is unserved. Eq. (8) shows that the capacity constraint is obeyed.

$c_{n}=c, \min (\|c-v\|) \wedge l_{c e}=1 \wedge l_{v e}=1 \wedge q_{g}=$ $1 \wedge s_{c}=0$

$q_{g}=\left\{\begin{array}{l}1, q_{\text {vnow }}+q_{c} \leq q_{v} \\ 0, q_{\text {vnow }}+q_{c}>q_{v}\end{array}\right.$

In the second routing process, the clusters are eliminated. Now, all unserved customers belong to their depot and are not partitioned by clusters. It means all vehicles can serve any unserved customers in the depot. The algorithm of the second routing is as follows.

1. A customer searches for the nearest vehicle in the depot that still has enough space in its storage to serve its request.

2. If there exists available vehicle, then this vehicle goes to this customer and pickups this request.

3. Step 1 and step 2 are repeated until all customers in the depot are scanned.

4. All vehicles return to the depot.

This algorithm is formalized by using Eq. (9). Eq. (9) shows that the selected vehicle is the nearest 
vehicle to the unserved customer and both vehicle and customer are in the same depot.

$$
v_{s}=v, \min (\|c-v\|) \wedge l_{v d}=1 \wedge l_{c d}=1 \wedge q_{g}=
$$$$
1
$$

\section{Simulation, result, and discussion}

This proposed model is then implemented into CVRP simulation so that its performance can be analyzed. In it, there are two observed variables. The first variable is total travel distance. The second variable is total unserved customers. The total travel distance is a commonly observed variable in many VRP studies. Meanwhile, the total number of unserved customers is introduced to evaluate the effectiveness of the routing process, especially in serving customers in a single routing process due to the limited number of vehicles and limited vehicle capacity.

There are four models from the previous works, as they are explained in the related works section, that are used as performance comparison. The first model is a hybrid evolutionary algorithm (HEA) [9]. The second model is partition-based algorithmnearest neighbor (PBA-NN) technique [8]. The third model is a hybrid genetic algorithm and nearest neighbor (GA-NN) [5]. The fourth model is simulated annealing (SA) [4]. These four techniques are chosen based on several theoretical reasons.

The HEA model [9] is the combination between metaheuristic approach (stochastic optimization) and deterministic approach. In this model, evolutionary algorithm is chosen as the metaheuristic part. The EA is a population-based optimization that adopts evolution system [29]. The quality of solution is improved during reproduction [29]. EA is used to determine the first customer for every vehicle [9]. After the first customer is determined, the next customers are selected by using nearest neighbor algorithm. The usage of the nearest neighbor is chosen based on its simplicity and its less computational consuming [9].

In the PBA-NN model [8], generally, all customers are partitioned so that every customer is clustered to the nearest depot. But, for the in-theborderline customer, it is clustered to the most available depot that is near to it. Like the HEA model [9], in the PBA-NN model [8], the nearest neighbor algorithm is used for routing process. The PBA-NN is full deterministic model.

In the third model, the genetic algorithm (GA) is combined with the nearest neighbor algorithm. Like the HEA model [9], this third model also combine the metaheuristic approach and the deterministic approach. This third model adopted the cluster-first route-second approach [5]. The customers are allocated to the nearest available depot / truck [5]. Meanwhile, the genetic algorithm is used in the routing process [5]. GA is a derivative of the EA [29]. Basically, the GA is proven as a flexible and simple solution for combinatorial optimization. In this third model, the GA is improved by embedding the nearest neighbor algorithm to reduce the randomness and the total travel distance by assigning the customers to the nearest possible trucks [5].

In the fourth model, the simulated annealing is chosen as a representative for full metaheuristic approach [4]. This method runs based on the thermodynamical principle [29]. Rather than other metaheuristic techniques, for example GA, the simulated annealing has advantage in focusing on global optimization [29]. This method can avoid the local optimal by accepting the current worse solution based on the stochastic requirements, the fitness gap between the current solution and the best solution so far, and the current temperature [29].

Based on this explanation, in this simulation, the proposed model (SM-K-NN) will be compared with three model types. The first type is full deterministic model which is represented by the PBA-NN [8]. The second type is the combined deterministic and metaheuristic models which is represented by the GA-NN [5] and HEA [9]. The third type is full metaheuristic model which is represented by the SA [4]. The deterministic model provides less computational solution. Meanwhile, the metaheuristic promises the improvement of the solution during the iteration or reproduction.

In the simulation, Surabaya city in Indonesia is adopted. Its size is 326 square kilometers. There are 5 depots which their location is distributed randomly, and it follows uniform distribution. There are 30 identical vehicles. Their maximum capacity is 40 lots. There are customers where their location is also distributed randomly, and it follows a uniform distribution. The average requested quantity for every customer is 10 lots. The actual requested quantity is generated randomly, and it follows a normal distribution. In this simulation, the adjusted variable is the number of customers. It ranges from 50 to 100 customers. The step size is 5 customers. For every number of customers, there are 30 simulation sessions. The result is shown in Fig. 4 to Fig. 7. Fig. 4 shows the relation between the number of customers and the number of unserved customers. Fig. 5 shows the relation between the number of customers and the service level percentage which is acquired by dividing the number of served customers and the number of total customers. Fig. 6 shows the 


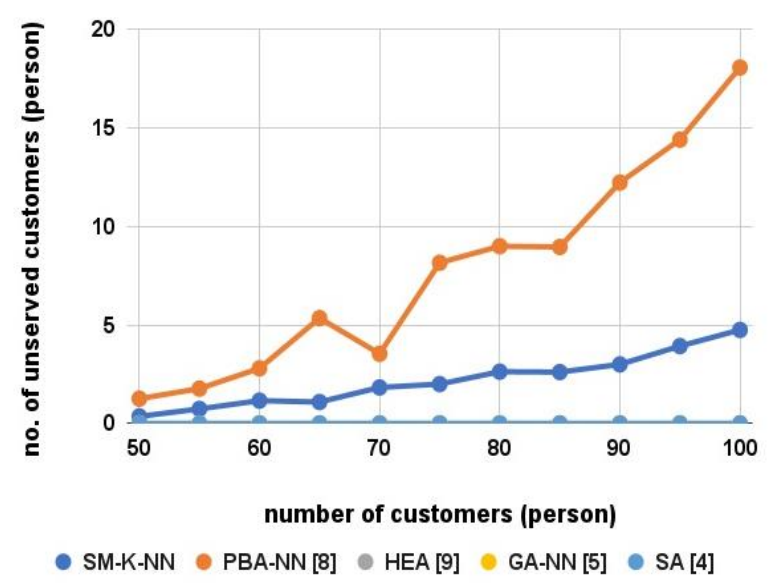

Figure. 4 Relation between the number of customers and the number of unserved customers

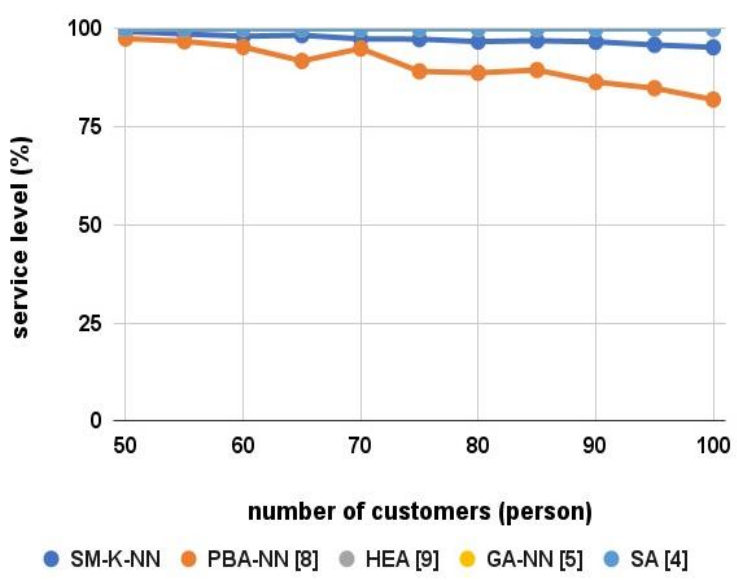

Figure. 5 Relation between the number of customers and the service level

relation between the number of customers and the total travel distance. Fig. 7 shows the relation between the number of customers and the average travel distance. The average travel distance is acquired by dividing the total travel distance and the total number of served customers.

Fig. 4 shows that the increasing of the number of customers makes the number of unserved customers increase too in the proposed model and the PBA-NN model [8]. Meanwhile, the HEA [9], GA-NN [5], and SA [4] models create a zero number of unserved customers. Compared between the proposed model and the PBA-NN model, the gap becomes wider due to the increasing of the number of customers. In the beginning, the proposed model creates 71 percent lower than the PBA-NN model [8] in the number of unserved customers aspect. When the number of customers is high (100 customers), the proposed model creates 73 percent lower in the number of unserved customers aspect.

Fig. 5 shows that in the proposed model and the PBA-NN model [8], the service level decreases due to the increasing of the number of customers.

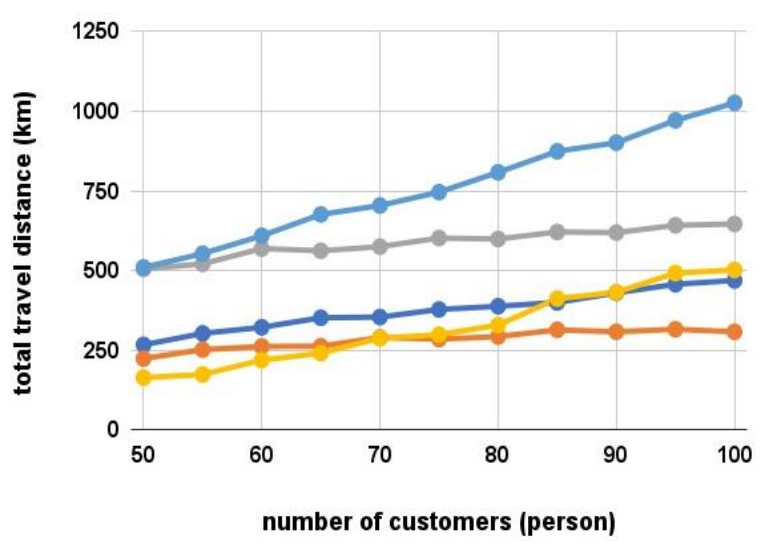

- SM-K-NN • PBA-NN [8] $\bullet$ HEA [9] GA-NN [5] $\bullet$ SA [4]

Figure. 6 Relation between the number of customers and total travel distance

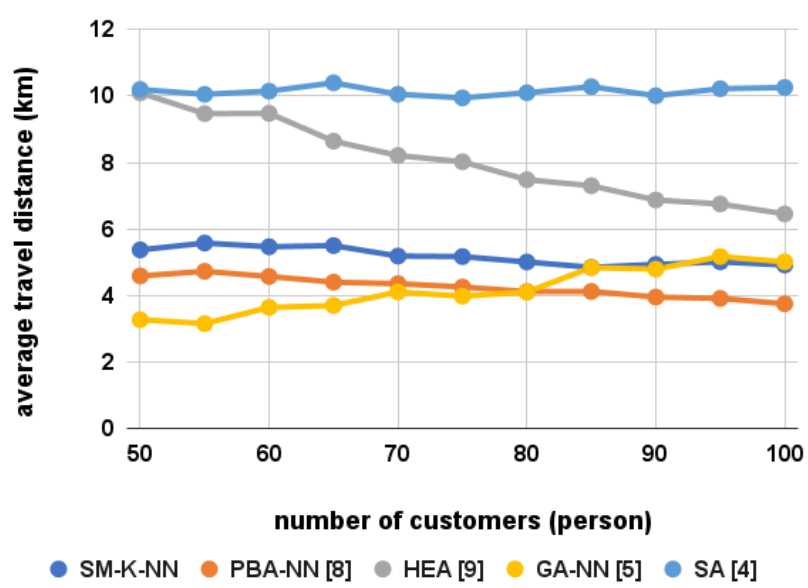

Figure. 7 Relation between the number of customers and average travel distance

Meanwhile, the HEA model [9], GA-NN model [5], and SA model [4] perform as the best model by creating 100 percent service level. Compared with the PBA-NN model [8], the proposed model performs better in the service level aspect. In the beginning, when the number of customers is 50 persons, the proposed model creates 1.8 percent higher than the PBA-NN model [8]. When the number of customers is high (100 persons), the proposed model creates 16.2 percent higher than the PBA-NN model [8]. It means that the gap between the proposed model and the PBA-NN model [8] in the service level aspect is wider due to the increasing of the number of customers.

Fig. 6 shows that the total travel distance increases due to the increasing of the number of customers. It happens in all models. In the total travel distance aspect, the PBA-NN model [8] performs as the best model by creating the lowest total travel distance when the number of customers is middle to high. On the other side, the GA-NN [5] 
performs as the best model when the number of customers is low to middle. On the other side, the SA model [9] performs as the worst model by creating the highest total travel distance. In the beginning, the proposed model creates 63 percent higher than the GA-NN model [5] and 48 percent lower than the SA model [4]. When the number of customers is high (100 customers), the proposed model creates 52 percent higher than the PBA-NN model [8] and 54 percent lower than the SA model [4].

Fig. 7 shows that the average travel distance decreases due to the increasing of the number of customers in the proposed model, HEA model [9], and PBA-NN model [8]. Meanwhile, the average travel distance tends to be stagnant in the SA model [4] and increases in the GA-NN model [5]. Compared among models, the PBA-NN model [8] performs as the best model by creating the lowest average travel distance when the number of customers is middle to high, and the GA-NN model [5] performs as the best model by creating the lowest average travel distance when the number of customers is low to middle. On the other side, the SA model [9] performs as the worst model by creating the highest average travel distance. The proposed model acts moderately. In the beginning, the proposed model creates 64 percent higher than the GA-NN model [5] and 47 percent lower than the SA model [4]. When the number of customers is high (100 customers), the proposed model creates 31 percent higher than the PBA-NN model [8] and 52 percent lower than the SA model [4].

There are several findings due to the simulation result. The proposed model becomes the trade-off model between the best model in creating the lowest total travel distance and the best model in creating the highest service level. The PBA-NN model [8] is proven in achieving the lowest total travel distance by sacrificing the unserved customers. On the other side, the SA model [9] is proven in achieving the highest service level by sacrificing total distance. In these circumstances, the proposed model plays moderately.

The cluster-first route-second policy [12], as it is conducted in the proposed model and the PBA-NN model [8] is proven in making these models can achieve low total travel distance. The clustering process makes the routing process focus on the customers that are near it. The PBA-NN model [8] conducts tighter clustering process by concerning mostly on the distance aspect. That is why its total travel distance is better than the proposed model. Meanwhile, by concerning the depot capacity too rather than the distance only, the proposed model creates lower number of unserved customers than the
PBA-NN model. Although the PBA-NN also concerns with the capacity only for the borderline customers, it shows that this population is not significant.

On the other side, global searching that is conducted in the HEA [9] and SA [4] is proven better in achieving the highest service level, i.e., the lowest number of unserved customers. Global searching policy makes the vehicle can search broader rather than local search approach in the cluster-first route-second policy. Unfortunately, this global search policy creates consequences in high total travel distance. It is because the vehicle will search any potential customer in the environment wherever it is possible, although sometimes it is far from the vehicle's current location.

\section{Conclusion}

This work has demonstrated that the combination between the stable marriage model and the K-means clustering algorithms is competitive enough to solve the capacitated vehicle routing problem (CVRP). It can compete the metaheuristic-based solution, which is in this work is a hybrid evolutionary algorithm, simulated annealing, and genetic algorithm-nearest neighbor. This work also shows that the unserved customer rate is an important aspect in evaluating the CVRP performance besides the common total travel distance aspect. It also demonstrates that the proposed model can be a trade-off between the total travel distance aspect and the number of unserved customers. In both aspects, the proposed model performs moderately between the compared models, the simulated annealing and the partition-based algorithm. In the number of unserved customers aspect, when the number of customers is low, the proposed model creates 71 percent lower than the PBA-NN model. Meanwhile, when the number of customers is high, the proposed model creates 73 percent lower than the PBA-NN model. In the total travel distance aspect, in the beginning, the proposed model creates 63 percent higher than the GA-NN model [5] and 48 percent lower than the SA model [4]. When the number of customers is high (100 customers), the proposed model creates 52 percent higher than the PBA-NN model [8] and 54 percent lower than the SA model [4]. In the average travel distance aspect, in the beginning, the proposed model creates 64 percent higher than the GA-NN model [5] and 47 percent lower than the SA model [4]. When the number of customers is high (100 customers), the proposed model creates 31 percent higher than the PBA-NN model [8] and 52 percent lower than the SA model [4]. 
This work indicates that the distribution of the vehicles plays important role in the performance of the multi-depot capacitated vehicle routing problem. In this work, the distribution of the vehicles is predetermined. In the future, study in distributing vehicles among depots is challenging, especially to meet the dynamic and stochastic demand. The circumstance whether the interaction among depots is coordinated or autonomously (collaborative or competitive), is also another interesting study.

\section{Conflicts of interest}

The authors declare no conflict of interest.

\section{Author contributions}

Conceptualization: Kusuma; methodology: Kusuma; software: Kusuma; validation: Kusuma and Kallista; analysis: Kusuma and Kallista; writing-original draft preparation: Kusuma; writingreview and editing: Kusuma and Kallista.

\section{Acknowledgments}

This work is supported by Telkom University.

\section{References}

[1] A. K. M. F. Ahmed and J. U. Sun, "Bilayer Local Search Enhanced Particle Swarm Optimization for Capacitated Vehicle Routing Problem", Algorithms, Vol. 11, article ID: 31, pp. 1-22, 2018.

[2] K. Braekers, K. Ramaekers, and I. van Nieuwenhuyse, "The Vehicle Routing Problem: State of the Art Classification Review", Computer \& Industrial Engineering, Vol. 99, pp. 300-313, 2016.

[3] L. Bertazzi and N. Secomandi, "Faster Rollout Search for the Vehicle Routing Problem with Stochastic Demands and Restocking", European Journal of Operation Research, Vol. 270, No. 2, pp. 487-497, 2018.

[4] N. M. E. Normasari, V. F. Yu, C. Bachtiar, and Sukoyo, "A Simulated Annealing Heuristic for the Capacitated Green Vehicle Routing Problem", Mathematical Problems in Engineering, article ID: 2358258, pp. 1-19, 2019.

[5] B. Khaoula, B. Youssef, and B. Ghizlane, "Multi-depots Vehicle Routing Problem with Simultaneous Delivery and Pickup and Inventory Restrictions: Formulation and Resolution", International Journal of Advanced Computer Science and Application, Vol. 10, No. 2, pp. 110-120, 2019.
[6] A. Annouch and A. Bellabdaoui, "An Adaptive Genetic Algorithm for a New Variant of the Gas Cylinders Open Split Delivery and Pickup with Two-dimensional Loading Constrains", International Journal of Advanced Computer Science and Application, Vol. 12, No. 1, pp. 607-619, 2021.

[7] Z. J. Peya, M. A. H. Akhnand, T. Sultana, and M. M. H. Rahman, "Distance Based Sweep Nearest Algorithm to Solve Capacitated Vehicle Routing Problem", International Journal of Advanced Computer Science and Application, Vol. 10, No. 10, pp. 259-264, 2019.

[8] W. Zhang, Y. Gajpal, S. S. Appadoo, and Q. Wei, "Multi-depot Green Vehicle Routing Problem to Minimize Carbon Emissions", Sustainability, Vol. 12, article ID: 3500, pp. 119, 2020.

[9] B. Peng, L. Wu, Y. Yi, and X. Chen, "Solving the Multi-depot Green Vehicle Routing Problem by a Hybrid Evolutionary Algorithm", Sustainability, Vol. 12, article ID: 2127, pp. 119, 2020.

[10] J. Lin, W. Zhou, O. Wolfson, "Electric Vehicle Routing Problem", Transportation Research Procedia, Vol. 12, pp. 508-521, 2016.

[11] S. Shao, W. Guan, B. Ran, Z. He, and J. Bi, "Electric Vehicle Routing Problem with Charging Time and Variable Travel Time", Mathematical Problems in Engineering, article ID: 5098183, pp. 1-13, 2017.

[12] M. Okulewicz and J. Mandziuk, "The Impact of Particular Components of the PSO-based Algorithm Solving the Dynamic Vehicle Routing Problem", Applied Soft Computing, Vol. 58, pp. 586-604, 2017.

[13] I. D. R. Cuevas, S. Omar, C. Morales, J. L. M. Flores, and J. R. M. Vazquez, "Capacitated Vehicle Routing Problem Model for Carriers", Journal of Transport and Supply Chain Management, Vol. 12, article ID: 345, pp. 1-9, 2018.

[14] X. Ge, Z. Zhu, and Y. Jin, "Electric Vehicle Routing Problems with Stochastic Demands and Dynamic Remedial Measures", Mathematical Problems in Engineering, article ID: 8795284, pp. 1-15, 2020.

[15] U. Yildirim and Y. Kuvvetli, "Solution of Capacitated Vehicle Routing Problem with Invasive Weed and Hybrid Algorithms", International Journal of Industrial Engineering Computations, Vol. 12, pp. 441-456, 2021.

[16] K. Ouaddi, Y. Benadada, and F. Z. Mhada, "Ant Colony System for Dynamic Vehicle Routing Problem with Overtime", International 
Journal of Advanced Computer Science and Application, Vol. 9, No. 6, pp. 306-315, 2018.

[17] Q. Qiao, F. Tao, H. Wu, X. Yu, and M. Zhang, "Optimization of a Capacitated Vehicle Routing Problem for Sustainable Municipal Solid Waste Collection Management Using the PSO-TS Algorithm", International Journal of Environment Research and Public Health, Vol. 17, article ID: 2163, pp. 1-22, 2020.

[18] A. I. Alhujaylan and M. I. Hosny, "A GRASPbased Solution Construction Approach for the Multi-vehicle Profitable Pickup and Delivery Problem", International Journal of Advanced Computer Science and Application, Vol. 10, No. 4, pp. 111-120, 2019.

[19] H. Jahangir, M. Mohammadi, S. H. R. Pasandideh, and N. Z. Nobari, "Comparing Performance of Genetic and Discrete Invasive Weed Optimization Algorithms for Solving the Inventory Routing Problem with an Incremental Delivery", Journal of Intelligent Manufacturing, Vol. 30, pp. 2327-2353, 2019.

[20] D. Gale and L. S. Shapley, "College Admissions and the Stability of Marriage", The American Mathematical Monthly, Vol. 69, No. 1, pp. 9-15, 1962.

[21] A. Abdulkadiroglu, Y. Che, and Y. Yasuda, "Expanding Choice in School Choice", American Economic Journal: Microeconomics, Vol. 7, No. 1, pp. 1-42, 2015.

[22] R. W. Irving, D. Manlove, and S. Scott, "The Hospitals/residents Problem with Ties", In: Proc. of the $7^{\text {th }}$ Scandinavian Workshop on Algorithm Theory, Norway, 2000.

[23] R. Bai, J. Li, J. A. D. Atkin, and G. Kendall, “A Novel Approach to Independent Taxi Scheduling Problem Based on Stable Matching", Journal of the Operational Research Society, Vol. 65, pp. 1501-1510, 2014.

[24] M. Kummel, F. Busch, and D. Z. W. Wang, "Taxi Dispatching and Stable Marriage", Procedia Computer Science, Vol. 83, pp. 163170, 2016.

[25] P. D. Kusuma and N. Rachmaningrum, "Higher Education Clustering in Indonesia by Using Kmeans and Geographical Similarity Methods", Journal of Engineering and Applied Sciences, Vol. 14, No. 19, pp. 7193-7209, 2019.

[26] C. Wu, B. Yan, R. Yu, B. Yu, X. Zhou, Y. Yu, and N. Chen, "K-means Clustering Algorithm and Its Simulation Based on Distributed Computing Platform", Complexity, Vol. 2021, article ID: 9446653, pp. 1-10, 2021.

[27] R. W. Irving, P. Leather, and D. Gusfield, "An Efficient Algorithm for the Optimal Stable
Marriage", Journal of the ACM, Vol. 34, No. 3, 1987.

[28] S. S. Shwartz and S. Ben-David, Understanding Machine Learning: From Theory to Algorithms, Cambridge University Press, New York, 2014.

[29] A. P. Engelbrecht, Computational Intelligence: An Introduction, $2^{\text {nd }}$ ed, Wiley, West Sussex, 2007. 